\title{
Differentiated thyroid carcinoma arising from or associated with struma ovarii: report of 2 cases
}

\section{Lavinia Magdalena VIJA, Andrei Goldstein, Daniela Neamtu, Corin Badiu, Mariana Purice \\ “C.I. Parhon" National Institute of Endocrinology, Bucharest, Romania}

\section{OBJECTIVES}

-Struma ovarii is a rare condition which elicited considerable interest because of its many unique features like its relationship to teratoma and differentiated thyroid cancer.

-The most common thyroid carcinomas to arise in struma ovarii are papillary and follicular.

-We describe two patients with differentiated thyroid carcinoma originating from malignant struma ovarii.

\section{Patient 1}

- 49 year old woman presenting with bone pain revealing follicular thyroid carcinoma metastases on biopsy

- Total thyroidectomy with lymphadenectomy revealed a follicular thyroid carcinoma with oxyphil component T3N1bM1.

-Two years later, ovarectomy revealed a malignant struma ovarii.

-Surgical resection of several vertebral metastases with spinal decompression and seriate adjuvant radioiodine therapy (10 GBq) were performed.

-WBS revealed high uptake on cervical lymph node and disseminated pulmonary and skeleton metastases justifying the pursue of radioiodine therapy.

\section{Patient 2}

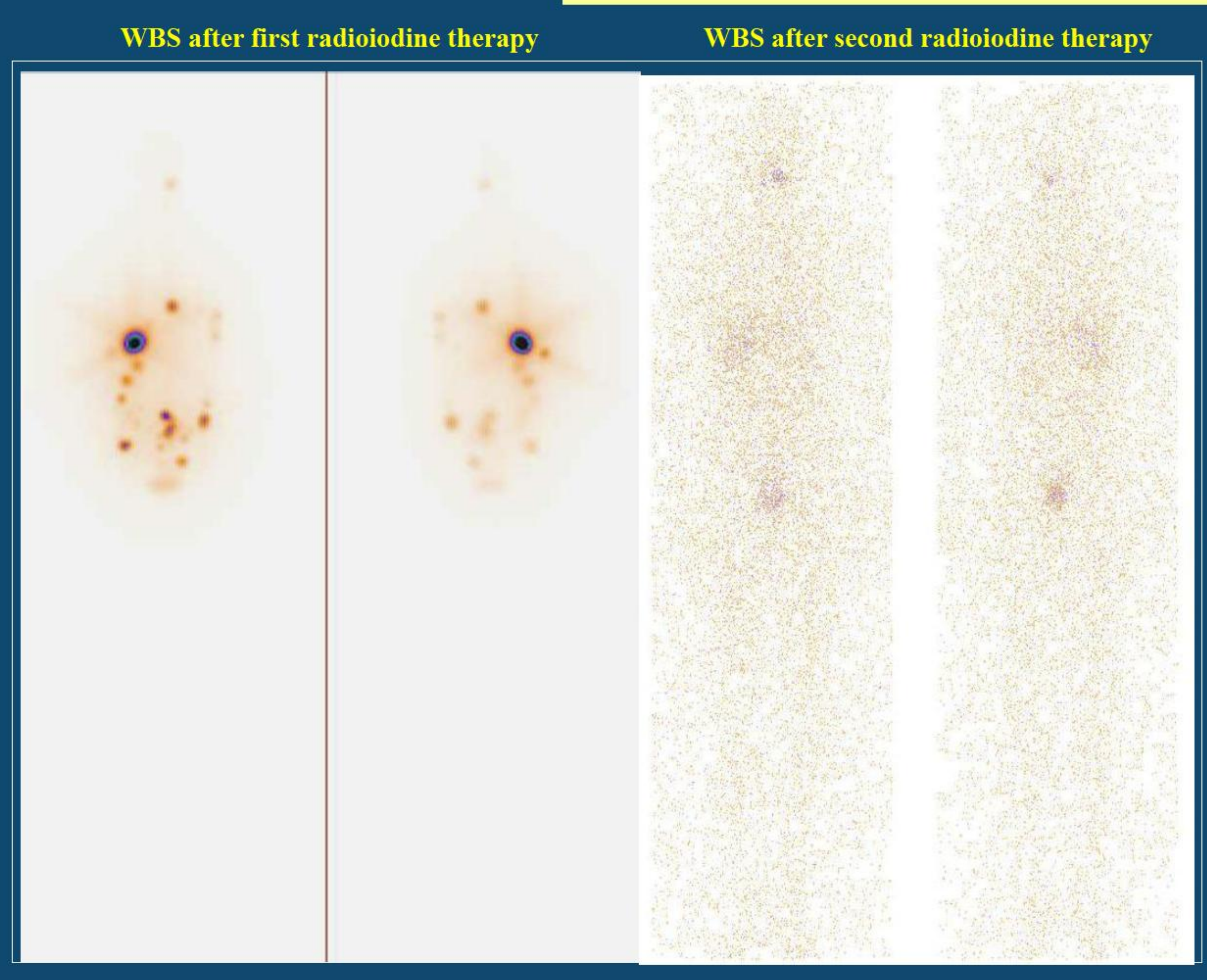

CONCLUSIONS

\section{- 32 year old woman}

- Ovarian teratoma with

-well differentiated follicular variant of papillary thyroid carcinoma

-peritoneal dissemination and appendix tumoral infiltration

- Two years later total thyroidectomy was performed (Hystology revealed chronic thyroiditis without thyroid cancer) Whole body scintigraphy (WBS) with therapeutic activity of I-

131 ( 2 sessions; cumulated activity: $11.1 \mathrm{GBq}$ ) revealed inital disseminated pulmonary and bone metastases with a complete response after radioiodine therapy, in agreement with undetectable Tg.

Radioiodine (RI) treatment characteristics

\begin{tabular}{l|l|l|l|l|l} 
I-131 (MBq) & Radioiodine & TSH (mU/L) & $\mathrm{Tg}$ (mg/dL) & ATG Ab & Whole body scan
\end{tabular} \begin{tabular}{l|l|l} 
Patient & Cumulated activity & Session No \\
\hline
\end{tabular} \begin{tabular}{|l|l|l|l|l|l|}
\hline Patient1 & 10000 & 3 & 75 & $>300$ negative & tro \\
\hline
\end{tabular} Mediastnay and lion

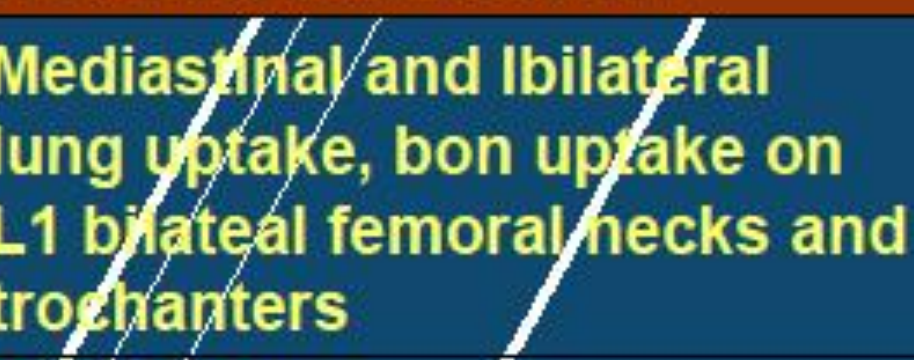
trogs (sseminated ytake within

100

2

